\title{
BMJ Open Interaction of ACE genotype and salt intake on hypertension among Chinese Kazakhs: results from a population- based cross-sectional study
}

\author{
Yuyan Wang, ${ }^{1}$ Biao Zhang, ${ }^{1}$ Lei Hou, ${ }^{1}$ Wei Han, ${ }^{1}$ Fang Xue, ${ }^{1}$ Yanhong Wang, ${ }^{1}$ \\ Yong Tang, ${ }^{2}$ Shaohua Liang, ${ }^{2}$ Weizhi Wang, ${ }^{2}$ Kuliqian Asaiti, ${ }^{3}$ Zixing Wang, ${ }^{1}$ \\ Yaoda Hu, ${ }^{1}$ Lei Wang, ${ }^{1}$ Changchun Qiu, ${ }^{1}$ Mingtao Zhang, ${ }^{2}$ Jingmei Jiang ${ }^{1}$
}

To cite: Wang Y, Zhang B, Hou L, et al. Interaction of ACE genotype and salt intake on hypertension among Chinese Kazakhs: results from a population-based crosssectional study. BMJ Open 2017;7:e014246. doi:10.1136/ bmjopen-2016-014246

YW and BZ contributed equally.

Received 20 September 2016 Revised 16 March 2017 Accepted 16 March 2017

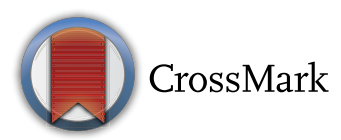

${ }^{1}$ Department of Epidemiology and Biostatistics, Institute of

Basic Medical Sciences, Chinese Academy of Medical Sciences, Beijing, China

${ }^{2}$ The People's Hospital in Altay Region, Xinjiang, China ${ }^{3}$ Hong Dun Town Hospital, Altay Region, Xinjiang, China

Correspondence to Dr Jingmei Jiang; jingmeijiang@ibms.pumc. edu.cn

\section{ABSTRACT}

Objectives To explore the effect of interaction between ACE genotype and salt intake on hypertension among Chinese Kazakhs, and to compare applications of interactions between logistic model and generalised partially linear tree-based regression (GPLTR) model. Design Population-based cross-sectional study.

Setting Hong Dun, North Xinjiang, China. Participants Non-consanguineous Chinese Kazakh participants ( $n=916,342$ men and 574 women) aged $\geq 30$ years.

Main outcome measures Association between ACE genotype and hypertension, association between salt intake and hypertension, and interaction of ACE genotype and salt intake on hypertension in two models.

Results Associations between salt intake and hypertension were different in ACE genotype of II and ID+DD. Under the logistic models, main and interaction effects were not observed for men, but effects were present in opposite directions for women (main effect of $\mathrm{ACE}: \mathrm{OR}=0.20$, $p=0.003$; interaction effect: $O R=1.07, p=0.027$ ). Under the GPLTR model, Bayesian information criterion trees included both salt intake and ACE genotype as split variables. Individuals with a salt intake $\geq 19.5 \mathrm{~g} /$ day and ID+DD genotypes had a 3.99-fold $(p=0.004)$ higher risk of hypertension compared with the II genotype for men, whereas salt intake $<20.1 \mathrm{~g} /$ day and ID+DD genotypes had an $\mathrm{OR}=0.55(\mathrm{p}=0.014)$ compared with the II genotype for women.

Conclusions An interaction of ACE genotype and salt intake on hypertension was observed among Chinese Kazakhs but in different ways according to sex. The GPLTR model appears to be more suitable for an exploration of interactions in complex diseases.

\section{INTRODUCTION}

Hypertension is a complex disorder resulting from an interaction of genetic and environmental factors. ${ }^{1}$ Blood pressure (BP) is regulated by the renin-angiotensin-aldosterone system (RAAS), ${ }^{2}$ which is heavily influenced by dietary salt intake. Indeed, dietary salt is recognised as the most important

\section{Strengths and limitations of this study}

- This is one of the few studies to explore the interaction of ACE genotype and salt intake on hypertension and is also the first such study in Chinese Kazakhs.

- The design strengths of the study include the extraction of participants without consanguinity, the use of stratified analyses to test the existence of an interaction effect and a comparison of the interaction exploration results from two models.

- The cross-sectional design cannot realise an investigation of the causal pathway of the association between the ACE genotype and salt intake interaction with hypertension, although possible mechanisms were suggested for the interaction effect on hypertension.

- Salt intake was assessed by urinary sodium excretion and might be underestimated because salt can be removed from the body through other means.

environmental contributor to hypertension. ${ }^{3}$ The most common genetic variation in RAAS is the insertion/deletion polymorphism of the ACE gene, ${ }^{4}$ and its association with BP has been reported to be dependent on sex and race/ethnicity. ${ }^{4-7}$ Although the presence of an interaction between ACE genotype and dietary salt intake has been supported by research findings, 89 few reports have investigated the effect of this interaction on hypertension in different populations. ${ }^{10}$

Measurements and explanations of candidate gene-environment interaction $(\mathrm{cG} \times \mathrm{E})$ in populations have long been explored, ${ }^{11}$ resulting in a general consensus that statistical concerns about their modelling are often overlooked. ${ }^{12}$ Popular logistic regression models including interaction terms can be problematic, ${ }^{1314}$ but the newly introduced generalised partially linear tree-based regression (GPLTR) model, which combines tree 
structure and linear adjustment, may offer more flexibility and power to the exploration of interactions in complex diseases. ${ }^{15}$

The aims of this study were to explore the interaction of ACE genotype and salt intake on hypertension among Chinese Kazakhs, who have a high prevalence of hypertension and a high dietary salt intake, and to compare the logistic model and the GPLTR model in interaction applications.

\section{METHODS}

\section{Study participants}

The design scheme, sampling process and data collection of the Chinese Xinjiang Altay Kazakh Heart Study have been presented elsewhere. ${ }^{1617}$ Hong Dun town is located in the urban rural fringe of Altay prefecture in North Xinjiang, China. From October 2012 to February 2013, a total of 1805 individuals aged $\geq 30$ years were recruited, and 1668 participants from 601 families completed the baseline survey and examination under standard procedures. Participants were required to have at least three paternal/maternal biological generations living in the same region without a history of intermarriage. Non-consanguineous spouses without consanguinity and only one member of biological relatives from each family were extracted to ensure independence (figure 1). Approval for the study was given by the Ethics Committee of the Institute of Basic Medical Sciences of the Chinese Academy of Medical Sciences, and written informed consent was obtained from all participants prior to enrolment.

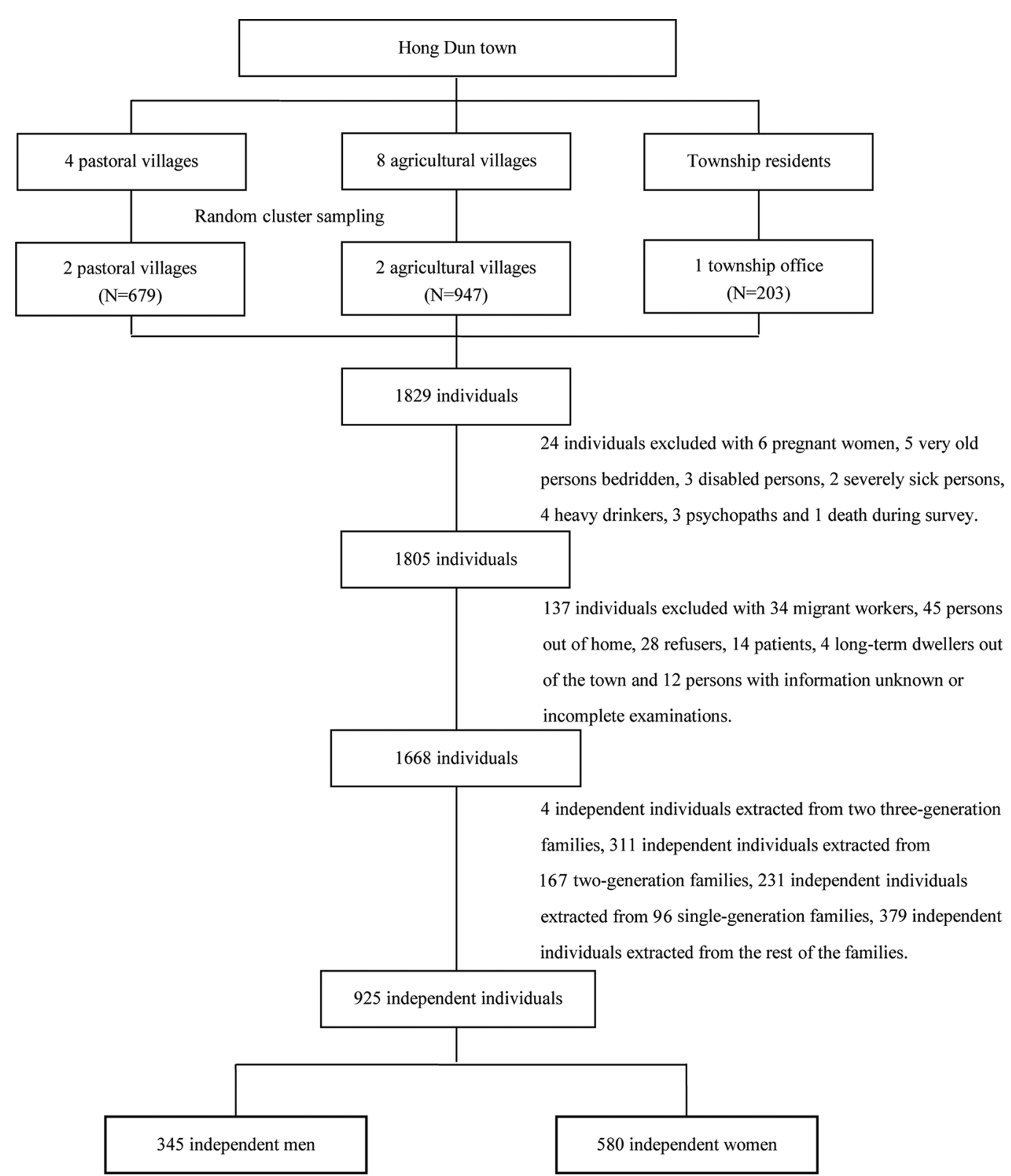

Figure 1 Flow chart for the 916 enrolled independent participants. 


\section{Study variables}

We complied with current Chinese Guidelines for Blood Pressure Measurement. ${ }^{18}$ A quiet room in a village clinic was selected for BP measurements, and all people in the room were required to keep quiet. Participants were comfortably seated, with legs uncrossed and the back and arm supported, such that the middle of the cuff on the upper arm was at the level of the right atrium (the mid-point of the sternum). BP was measured with an appropriate arm cuff and a mercury column sphygmomanometer on the left arm after a resting period of at least $10 \mathrm{~min}$ in the supine position. All measurements used the same standard procedure and calibrated mercury sphygmomanometers, and were conducted by trained and qualified staff. Currently, a large-size arm cuff for a mercury column sphygmomanometer is not available in the market of China, so radial artery auscultation on the upper forearm was used for obese individuals with large upper arms, as recommended by BP measurement guidelines. ${ }^{18} \mathrm{BP}$ was defined as the mean of two readings, or three readings if there was a difference of more than $5 \mathrm{~mm} \mathrm{Hg}$ between the initial readings. Participants were classified as hypertensive if they met at least one of the following three criteria: (1) a systolic $\mathrm{BP} \geq 140 \mathrm{~mm} \mathrm{Hg}$; (2) a diastolic $\mathrm{BP} \geq 90 \mathrm{~mm} \mathrm{Hg}$ and (3) the use of antihypertensive medications within the last 2 weeks.

Venous blood samples were drawn after an overnight fast of at least 10 hours and centrifuged within $20 \mathrm{~min}$, then divided into two. One sample was used for biochemistry assays performed within 4 hours, and the other was stored in a portable refrigerator at $-20^{\circ} \mathrm{C}$ then transferred as soon as possible to Beijing, where it was kept at $-80^{\circ} \mathrm{C}$ for genetic analysis. D and I ACE alleles were identified by PCR amplification of the respective fragments from ACE intron 16 and size fractionation and visualisation by electrophoresis. The ACE genotype was classified as II, ID or DD depending on the presence of each allele, with a $99.76 \%$ genotyping success rate.

The second urine sample after waking was collected, and urinary sodium was measured with a Caretium XI-921CT automatic electrolyte analyzer (Shenzhen, China). Salt intake was assessed by urinary sodium excretion per day from the amounts of urinary sodium, urine creatinine (Cr) and the 24 hours urinary Cr excreted as estimated from height, body weight and age ${ }^{19}$ for which each $43 \mathrm{mmol}$ of sodium is approximately equivalent to 1 $\mathrm{g}$ of sodium or $2.5 \mathrm{~g}$ of salt (sodium chloride).

Other covariates were also collected using standard procedures. Demographic information (including age, sex, occupation and education) and information about diet, alcohol consumption, cigarette smoking and medical history were obtained from a face-to-face questionnaire survey. Body weight and height were measured in light indoor clothes and without shoes. Body mass index (BMI) was calculated as body weight in kilograms divided by height in metres squared $\left(\mathrm{kg} / \mathrm{m}^{2}\right)$. Fasting blood glucose (FBG) was collected according to standardised protocols by a Beckman Coulter AU2700 clinical chemistry analyser (California, USA).

\section{GPLTR model and selection criteria}

$\mathrm{Y}$ was denoted as the outcome of interest, $\mathrm{X}$ were the confounding variables to be modelled linearly and $G$ were the group of risk factors whose interaction effect was to be approximated by the tree-based structure. The main purpose of this GPLTR model was to test the combined effect of $\mathrm{G}$ on $\mathrm{Y}$ while adjusting for the confoundersX; the model was expressed as

$\mathrm{g}(\mathrm{E}(\mathrm{YlX}, \mathrm{Z}))=\mathrm{X}^{\prime} \theta+\beta_{\mathrm{T}} \mathrm{F}(\mathrm{T}(\mathrm{Z}))$, where $\mathrm{g}($.$) is a$ specific link function (generalised linear model), such as the logit link function $g(\mu)=\log \left(\frac{\mu}{1-\mu}\right)$ for a binary outcome, and $\mathrm{F}(\mathrm{T}(\mathrm{Z}))$ is the vector of indicator variables signifying the leaves of the tree $\mathrm{T}(\mathrm{Z})$.

In this study, we used $\mathrm{Y}$ to represent the presence of hypertension, G to be the ACE genotype and the estimated salt intake and $\mathrm{X}$ to be the environmental risk factors having an apparent impact on the hypertension rate. First, we constructed a maximal tree with successive steps of initialization, iteration and ending conditions. The goodness of a candidate split was assessed for each node by the deviance of a generalised linear model, which equals the test deviance of the parent node minus the sum of the deviance of the two child nodes. Second, the sequence of nested candidate subtrees was created, and Akaike information criterion (AIC) and Bayesian information criterion (BIC) were used to determine the best tree in the sequence.

\section{Statistical analysis}

All analyses were conducted separately by sex. Basic characteristics were compared with regard to the status of hypertension (table 1). Variables significantly different between hypertension and normotension were chosen as confounders; therefore, smoking and family history of hypertension were not included as confounders in further analyses. ORs with 95\% CIs were calculated to assess the associations of the ACE polymorphism with hypertension (table 2). To examine the presence of an interaction, associations of salt intake with hypertension were illustrated as stratified by ACE genotype as II and ID+DD (table 3). To compare the effect of the interaction, two models were applied (table 4), a logistic regression model and a GPLTR model adjusting for the same covariates to extract the high-risk group of hypertension using AIC and BIC (figure 2). Description and preliminary analyses were carried out by SAS V.9.3 software, and GPLTR models were conducted by R V.3.1.3 with the 'GPLTR' package. One-sided hypotheses were established for trend tests based on the logistic model, and all $p$ values were two-tailed and considered significant at $\leq 0.05$.

\section{RESULTS}

This study enrolled 916 independent participants (342 men and 574 women) who had complete data for all 
Table 1 Basic characteristics of enrolled 916 participants by sex and hypertension status

\begin{tabular}{|c|c|c|c|c|c|c|}
\hline & \multicolumn{3}{|l|}{ Men $(n=342)$} & \multicolumn{3}{|l|}{ Women $(n=574)$} \\
\hline & $\begin{array}{l}\text { Hypertension } \\
(n=169)\end{array}$ & $\begin{array}{l}\text { Normotension } \\
(n=173)\end{array}$ & $p$ value & $\begin{array}{l}\text { Hypertension } \\
(n=206)\end{array}$ & $\begin{array}{l}\text { Normotension } \\
(\mathrm{n}=368)\end{array}$ & $p$ value \\
\hline Age, years & $50.1 \pm 12.1$ & $42.8 \pm 10.5$ & $<0.001$ & $51.0 \pm 11.2$ & $39.6 \pm 7.8$ & $<0.001$ \\
\hline \multicolumn{7}{|l|}{ Occupation, n (\%) } \\
\hline Nomad & $68(40.2)$ & $67(38.7)$ & 0.900 & 87 (42.2) & $114(31.0)$ & 0.001 \\
\hline Farmer & 75 (44.4) & $81(46.8)$ & & $94(45.6)$ & $169(45.9)$ & \\
\hline City worker & $26(15.4)$ & $25(14.5)$ & & 25 (12.2) & $85(23.1)$ & \\
\hline \multicolumn{7}{|l|}{ Education, n (\%) } \\
\hline Primary school and below & $61(36.1)$ & $40(23.1)$ & 0.028 & $75(36.4)$ & $63(17.1)$ & $<0.001$ \\
\hline Junior middle school & $69(40.8)$ & $89(51.5)$ & & $86(41.8)$ & $161(43.8)$ & \\
\hline High school and above & $39(23.1)$ & $44(25.4)$ & & $45(21.8)$ & $144(39.1)$ & \\
\hline $\mathrm{BMI}, \mathrm{kg} / \mathrm{m}^{2}$ & $26.9 \pm 4.8$ & $24.6 \pm 3.4$ & $<0.001$ & $28.7 \pm 5.4$ & $25.8 \pm 4.5$ & $<0.001$ \\
\hline Smoking, $\mathrm{n}(\%)$ & $116(69.8)$ & $133(76.9)$ & 0.140 & $12(5.8)$ & $11(3.0)$ & 0.097 \\
\hline Drinking, n (\%) & $53(31.4)$ & $38(22.0)$ & 0.049 & $0(0.0)$ & $0(0.0)$ & - \\
\hline $\mathrm{SBP}, \mathrm{mm} \mathrm{Hg}$ & $152.3 \pm 19.2$ & $122.2 \pm 9.5$ & $<0.001$ & $154.1 \pm 21.7$ & $117.8 \pm 9.8$ & $<0.001$ \\
\hline DBP, $\mathrm{mm} \mathrm{Hg}$ & $95.7 \pm 11.2$ & $77.9 \pm 7.2$ & $<0.001$ & $94.1 \pm 11.4$ & $75.5 \pm 7.7$ & $<0.001$ \\
\hline Family history of hypertension, $\mathrm{n}(\%)$ & $92(57.1)$ & $94(56.3)$ & 0.876 & $116(60.1)$ & 213 (58.2) & 0.663 \\
\hline FBG, $\mathrm{mmol} / \mathrm{L}$ & $5.4 \pm 0.8$ & $5.2 \pm 0.4$ & $<0.001$ & $5.3 \pm 0.8$ & $5.0 \pm 0.5$ & $<0.001$ \\
\hline Salt intake, $g /$ day & $19.5 \pm 26.7$ & $18.3 \pm 15.9$ & 0.599 & $17.1 \pm 7.4$ & $15.9 \pm 9.7$ & 0.129 \\
\hline
\end{tabular}

Values are means $\pm \mathrm{SD}$ and frequency (percent).

BMI, body mass index; DBP, diastolic blood pressure; FBG, fasting blood glucose; SBP, systolic blood pressure.

study variables. The prevalence of hypertension was $49.4 \%$ for men and $35.9 \%$ for women. Participant characteristics are shown in table 1 . Briefly, participants with hypertension had increased age, BMI and FBG compared with normotensive participants for both sexes.

\section{Independent associations}

The independent associations of the ACE genotype and salt intake with hypertension are shown in table 2. The D allele had a protective effect on hypertension in women, with the DD genotype having an $\mathrm{OR}=0.47$ (95\% CI 0.25 to 0.88 ) compared with the II genotype. This protective effect was not seen in men, with the DD genotype having an $\mathrm{OR}=1.27$ (95\% CI 0.66 to 2.46) compared with the II genotype. Salt intake by quartiles did not correlate with hypertension for either sex ( $p$ for trend=0.099 for men and 0.178 for women).

\section{Interaction effect}

\section{Stratified analysis}

Associations between salt intake and hypertension stratified by ACE II genotype and ID+DD genotypes were evaluated (table 3). No correlation was observed in the II genotype group for either men ( $p$ for trend=0.967) or women ( $\mathrm{p}$ for trend=0.904). An increase in hypertension with a higher salt intake was observed in the ID+DD genotypes group for both men ( $\mathrm{p}$ for trend=0.028) and women ( $\mathrm{p}$ for trend=0.045).

\section{Logistic mode}

Results from the logistic regression model adjusted for multiple confounders are shown in table 4 . For men, no main effects of the ACE genotype $(\mathrm{OR}=1.33, \mathrm{p}=0.426)$ or salt intake $(\mathrm{OR}=1.00, \mathrm{p}=0.415)$ were observed, and there was no interaction effect $(\mathrm{OR}=0.99, \mathrm{p}=0.464)$. For women, no main effect of salt intake $(\mathrm{OR}=0.99, \mathrm{p}=0.523)$ was observed, but a main effect of the ACE genotype $(\mathrm{OR}=0.20, \mathrm{p}=0.003)$ and an interaction effect $(\mathrm{OR}=1.07$, $\mathrm{p}=0.027)$ were observed to be present in opposite directions. Recessive model was also built as sensitivity analysis, and ORs were turned as reciprocals as expected, which confirmed the robustness of the findings.

\section{GPLTR model}

All iterative procedures converged after 15 iterations, and trees selected by AIC and BIC with different degrees of pruning resulted in 10 and 7 leaves for men, and 9 and 3 leaves for women, respectively (figure 2). BIC trees were chosen for the final interpretation based on their stability. Risk assessments of trees are shown in table 4. For men, leaves are identified from the left as

1. Node 1: 57 participants characterised by salt intake $\geq 19.5 \mathrm{~g} /$ day and ACE II genotype;

2. Node 2: 40 participants characterised by salt intake $\geq 19.5 \mathrm{~g} /$ day and ACE ID+DD genotype;

3. Node 3: 127 participants characterised by $15.3 \mathrm{~g} /$ day $\leq$ salt intake $<19.5 \mathrm{~g} /$ day; 


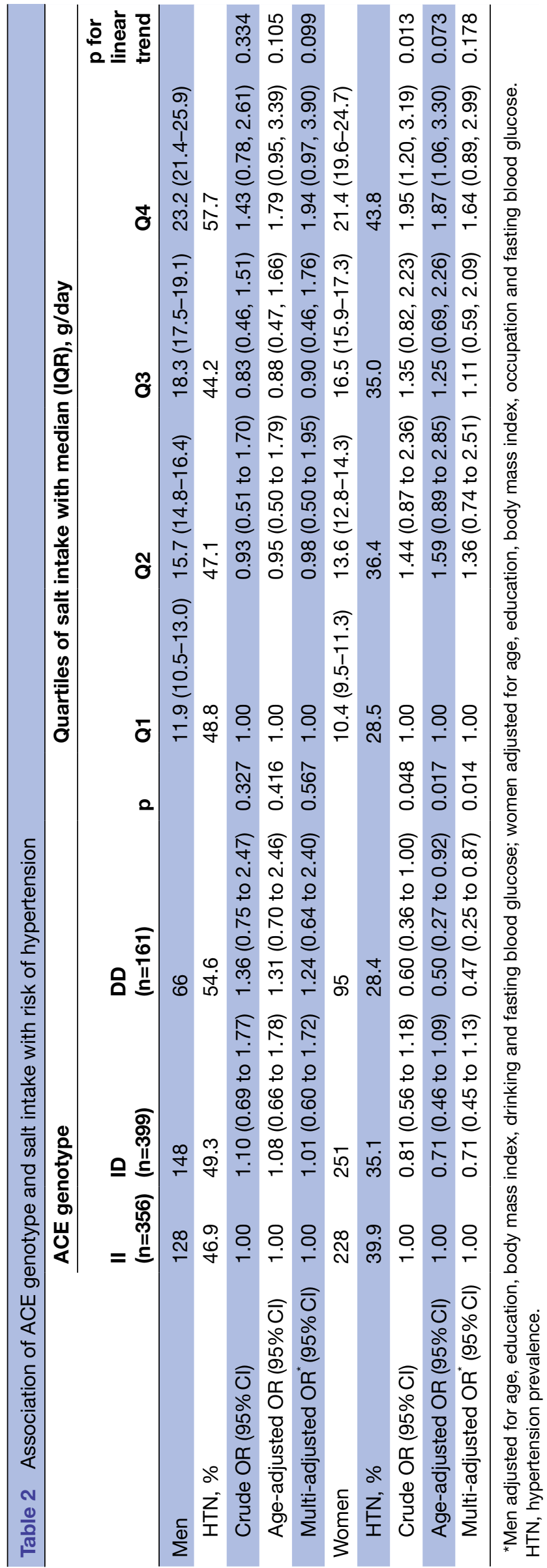

4. Node 4: 13 participants characterised by $14.6 \mathrm{~g} /$ day $\leq$ salt intake $<15.3 \mathrm{~g} /$ day;

5. Node 5: 57 participants characterised by $12.2 \mathrm{~g} /$ day $\leq$ salt intake $<14.6 \mathrm{~g} /$ day;

6. Node 6: 17 participants characterised by salt intake $<9.6 \mathrm{~g} /$ day;

7. Node 7: 31 participants characterised by $9.6 \mathrm{~g} /$ day $\leq$ salt intake $<12.2 \mathrm{~g} /$ day.

Split variables for the selected tree included both salt intake and ACE genotype as II and ID+DD. After adjusting for the same confounders in the multivariable logistic model, the final tree showed that Node 2 had 3.99-fold $(\mathrm{p}=0.004)$ higher risk for hypertension compared with Node 1 , and Node 3 had an OR=0.93 ( $\mathrm{p}=0.860)$. For women, leaves were identified from the left as

1. Node 1: 185 participants characterised by salt intake $<20.1 \mathrm{~g} /$ day and ACE II genotype;

2. Node 2: 288 participants characterised by salt intake $<20.1 \mathrm{~g} /$ day and ACE ID+DD genotype;

3. Node 3: 101 participants characterised by salt intake $\geq 20.1 \mathrm{~g} /$ day.

Split variables also included both salt intake and ACE genotype as II and ID+DD. Node 2 had an $\mathrm{OR}=0.55$ $(\mathrm{p}=0.014)$ and Node 3 had an OR=1.26 $(\mathrm{p}=0.444)$ compared with Node 1.

\section{DISCUSSION}

There are three main findings from the present study. First, we observed that the association between ACE gene polymorphism and hypertension was sex specific. Second, stratified analysis indicated that an interaction between ACE genotype and salt intake existed for both sexes. Third, an application comparison of the two models suggested that the traditional logistic model was not appropriate in this situation, and that the GPLTR model could effectively determine the high-risk characteristics of hypertension.

The distribution of the ACE genotype observed in this study is similar to that reported for an isolated group of Kazakhs in $2003,{ }^{20}$ with a DD genotype frequency of less than 20\%, which differs from that observed in other studies on Kazakhs ${ }^{21}$ and in studies of other ethnicities. ${ }^{4-7}$ This difference may arise from the diverse structure of the population and racial heterogeneity. Our current results are consistent with previous studies that reported ACE genotype to be a sex-specific candidate gene for hypertension. ${ }^{5622} 23$ No positive association was detected between the $\mathrm{D}$ allele and hypertension in men, but a protective effect of the $\mathrm{D}$ allele was observed for women, presumably through sexes, ethnicities and other factors including gene-gene or gene-environment interactions. ${ }^{6}$ A non-linear association between salt intake and risk of hypertension was confirmed by restricted cubic spline with automatically computed 'knots' (data not shown). With regard to the interaction of ACE genotype and salt intake on hypertension, a previous study that examined 284 Japanese men using logistic regression analysis ${ }^{10}$ 
Table 3 Multivariable-adjusted ORs (95\% Cl) in the ACE genotypes of II and ID+DD by different salt intake levels in men and women

\begin{tabular}{llllll}
\hline \multirow{2}{*}{$\begin{array}{l}\text { Multi-adjusted } \\
\text { OR }^{*}(\mathbf{9 5 \%} \text { Cl) }\end{array}$} & Q1 & Q2 & Q3 & Q4 & $\begin{array}{l}\text { p for linear } \\
\text { trend }\end{array}$ \\
\hline Men & $11.9(10.5-13.0)$ & $15.7(14.8-16.4)$ & $18.3(17.5-19.1)$ & $23.2(21.4-25.9)$ & \\
\hline II $(n=128)$ & 1.00 & $2.04(0.66-6.30)$ & $1.59(0.51-5.01)$ & $1.09(0.37-3.19)$ & 0.967 \\
ID+DD $(n=214)$ & 1.00 & $0.59(0.25-1.41)$ & $0.68(0.30-1.58)$ & $3.15(1.25-7.93)$ & 0.028 \\
Women & $10.4(9.5-11.3)$ & $13.6(12.8-14.3)$ & $16.5(15.9-17.3)$ & $21.4(19.6-24.7)$ & \\
\hline II $(n=228)$ & 1.00 & $1.19(0.47-2.99)$ & $0.75(0.28-2.04)$ & $1.15(0.44-3.00)$ & 0.904 \\
\hline ID+DD $(n=346)$ & 1.00 & $1.55(0.67-3.59)$ & $1.53(0.66-3.52)$ & $2.35(1.06-5.24)$ & 0.045 \\
\hline
\end{tabular}

*Men adjusted for age, education, body mass index, drinking and fasting blood glucose;

women adjusted for age, education, body mass index, occupation and fasting blood glucose.

reported a negative association for ACE (II+ID vs DD: $\mathrm{OR}=0.1, \mathrm{p}=0.024)$ and a positive association for interaction [ACE (II+ID vs DD) $\times$ salt intake level: $\mathrm{OR}=3.6$, $\mathrm{p}=0.047]$ in opposite directions, which was similar to our current results when the II genotype was set as the reference.

Strong evidence from association and linkage studies suggests that ACE has a profound effect on the variance of plasma ACE levels, ${ }^{24}$ which maintain BP homeostasis. Among the major factors that activate RAAS, dietary sodium/potassium balance is the most important and is mainly influenced by dietary salt intake. ${ }^{3}$ The sodium status has been reported to affect the ACE I/D polymorphism phenotype, and to enhance $\mathrm{BP}$ and renal function responses, while the conversion of aldosterone to angiotensin I with the DD genotype is blunted by low a sodium intake. ${ }^{8}$ These reports suggest that the ACE polymorphism interacts with dietary salt intake, with subsequent effects on the regulation of BP. Gene-targeting experiments that inactivate ACE in mice have provided evidence for the sex-specific effect of ACE gene on blood pressure. ${ }^{25}$ Population-based research also supports the sex-specific influence of ACE on BP variability. ${ }^{426}$ Assessment of the dietary habits of individuals in this study (data not shown) revealed average salt intake of $17.3 \mathrm{~g} /$ day, which is far greater than that of other populations and WHO recommendations. ${ }^{27}$ Given the high daily salt intake for this population, we suggest that the potential harmful effects of high salt intake on the development of hypertension should be emphasised to Chinese Kazakhs. Previous studies into the relationship between ACE and salt sensitivity indicated possession of the ACE D allele by men may lead to an increased response to a high salt intake $^{428}$; thus, carriers of the D allele may trigger hypertension if they consume a high salt diet. For women, the mechanism behind the interaction is uncertain; however,

Table 4 ACE genotype and salt intake interaction explorations by logistic model and generalised partially linear tree-based regression model

\begin{tabular}{|c|c|c|c|c|c|}
\hline Logistic Model & & & GPLTR Model & & \\
\hline Terms in the model & $\begin{array}{l}\text { Multi-adjusted } \\
\text { OR }^{*}(95 \% \mathrm{Cl})\end{array}$ & $\begin{array}{l}\mathbf{p} \\
\text { value }\end{array}$ & Nodes in the model & $\begin{array}{l}\text { Multi-adjusted } \\
\text { OR }^{*}(95 \% \mathrm{Cl})\end{array}$ & $\begin{array}{l}p \\
\text { value }\end{array}$ \\
\hline Men $(\mathrm{ROC}=0.747)$ & & & Men $(\mathrm{ROC}=0.809)$ & & \\
\hline Salt intake, g/day & 1.00 (0.99 to 1.03$)$ & 0.415 & $\begin{array}{l}\text { Node 1: Salt intake } \geq 19.5 \text { and } \\
\text { ACE (II) }\end{array}$ & 1.00 & \\
\hline ACE (ID+DD vs II) & 1.33 (0.66 to 2.65$)$ & 0.426 & $\begin{array}{l}\text { Node 2: Salt intake } \geq 19.5 \text { and } \\
\text { ACE (ID+DD) }\end{array}$ & 3.99 (1.55 to 10.26$)$ & 0.004 \\
\hline ACE (ID+DD vs II) ×salt intake & 0.99 (0.96 to 1.02$)$ & 0.464 & Node 3: $15.3 \leq$ salt intake $<19.5$ & 0.93 (0.41 to 2.09$)$ & 0.860 \\
\hline Women $(\mathrm{ROC}=0.839)$ & & & Women $(\mathrm{ROC}=0.839)$ & & \\
\hline Salt intake, g/day & 0.99 (0.94 to 1.03$)$ & 0.523 & $\begin{array}{l}\text { Node 1: Salt intake }<20.1 \text { and } \\
\text { ACE (II) }\end{array}$ & 1.00 & \\
\hline ACE (ID+DD vs II) & 0.20 (0.07 to 0.59$)$ & 0.003 & $\begin{array}{l}\text { Node 2: Salt intake }<20.1 \text { and } \\
\text { ACE }(\text { ID+DD) }\end{array}$ & 0.55 (0.34 to 0.89$)$ & 0.014 \\
\hline ACE (ID+DD vs II) $\times$ salt intake & 1.07 (1.01 to 1.13$)$ & 0.027 & Node 3: $20.1 \leq$ salt intake & 1.26 (0.70 to 2.25$)$ & 0.444 \\
\hline
\end{tabular}

*Men adjusted for age, education, body mass index, drinking and fasting blood glucose; women adjusted for age, education, body mass index, occupation and fasting blood glucose.

GPLTR, generalized partially linear tree-based regression; ROC, receiver operating characteristic curve. 


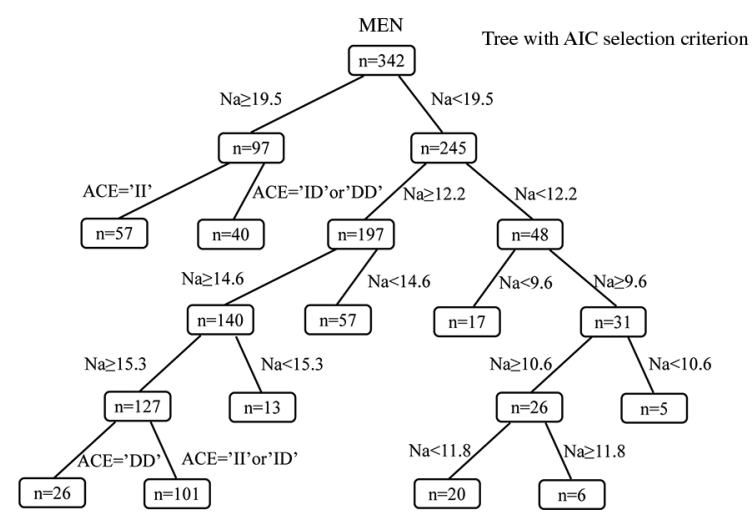

Tree with BIC selection criterion

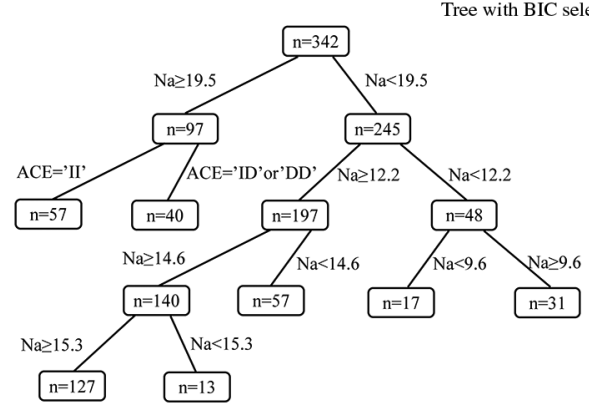

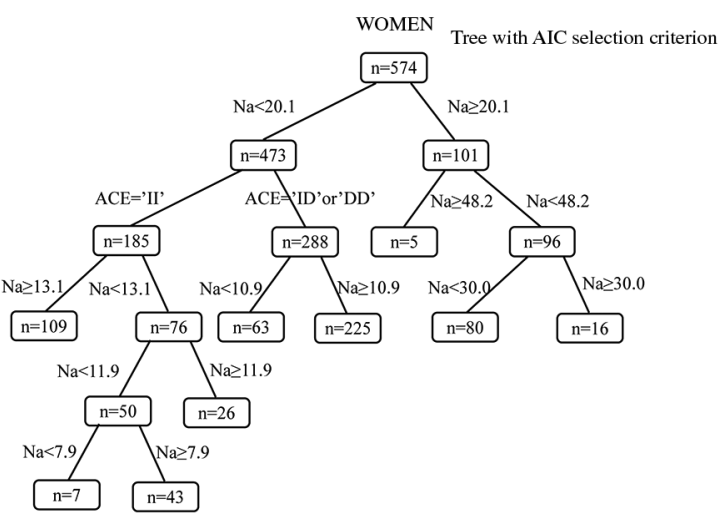

Tree with BIC selection criterion

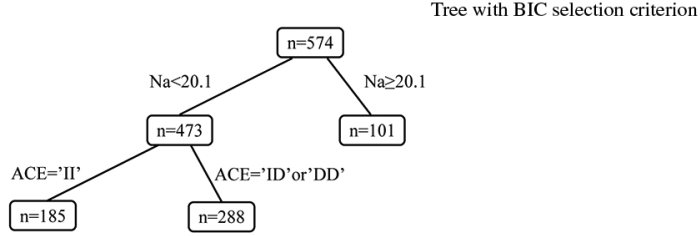

Figure 2 Optimal trees obtained from 342 men and 574 women using Akaike information criterion (AIC) and Bayesian information criterion $(\mathrm{BIC})$. Leaves are denoted by rectangles and the number in each node represents the number of participants falling in this leaf.

the combination of the ACE D allele and a lower salt intake may have a synergistic effect on hypertension. ${ }^{28}$

Previous discussions into gene-environment interactions have historically progressed through three main stages, including eugenics, IQ and the serotonin transporter gene controversy. ${ }^{29}$ This has resulted in an apparent divide between variation-partitioning and mechanism-elucidation approaches. The former approach interpreted interactions as strictly statistical phenomena, as suggested by Fisher, ${ }^{11}$ while the latter emphasised the need to probe and understand the causal mechanisms behind interaction responsible for the developmental process, as suggested by Hogben. ${ }^{11}$ For complex diseases like hypertension, failure to pay proper attention to the methodological checks that must be undertaken for $\mathrm{cG} \times \mathrm{E}$ has resulted in some studies making dubious claims. ${ }^{30}{ }^{31}$ Logistic regression requiring main effects and linear conditions is usually restricted to low-order interactions, and exploration relying on cross-product terms to measure the cause of the variation responsible for the interaction can be particularly problematic. We have built a full model using three main factors (ie, gender, ACE genotype and salt intake) in which the three main effects and the second-order interaction term are positive, but the three first-order interaction terms are all negative. Moreover, explanatory and confounding variables are entered equally into logistic regression models, which may disperse or conceal the actual main and interactive effects. ${ }^{32}$ For example, the main protective effects from the ACE genotype and the risk interaction effects of the
ACE genotype and salt intake in women of the current study cannot be readily explained because the ACE genotype has no reported independent effect on hypertension and rarely shows the opposite effect after interacting with other factors. ${ }^{8}$ Therefore, new models suitable for complicated high-order interactions and appropriate confounder adjustments, designed to be distinguished from generalised linear regression models (GLMs), are warranted to reveal gene-environment interactions for complex diseases. ${ }^{33}$ The GPLTR model is semi-parametric and integrates the advantages of the tree structure and GLMs. Its purpose is to decompose a data space recursively into smaller areas defined by a set of explanatory variables with adjustment for confounding variables, ${ }^{15} 3435$ where explanatory and confounding variables are not treated in the same way. The modified procedure ${ }^{15}$ can explicitly identify subgroups of individuals at high risk for hypertension with fewer computational demands, where highlighted features of subgroup provide clues for the mechanisms of disease development. Taking the results of the GPLTR model in the current study as an example, the ACE D allele was suggested to be the trigger for hypertension among men with a high salt intake, and female carriers of the $\mathrm{D}$ allele combined with a lower salt intake may offer help protection from the development of hypertension. Our calculation of the 10-fold cross-validation prediction error (data not shown) of the logistic regression model and GPLTR model for both men and women revealed the overall improved accuracy of GPLTR model. 
The high-quality study design, a response rate of $92.4 \%$ and high aggregation of Chinese Kazakhs in this study provide good internal validity of the results. Sex-stratified analyses were performed to reveal potential differences in mechanisms behind the effect of the ACE genotype and salt intake interaction on hypertension. The high prevalence of hypertension $(49.4 \%$ for men and $35.9 \%$ for women) allowed for robust comparisons between individuals with hypertension and non-hypertensive controls. Application comparisons between the logistic model and the GPLTR model was based on the same confounding variables by sex, which were cautiously chosen according to baseline results. The study should also be considered in light of its limitations. The cross-sectional design cannot realise investigation of the causal pathway of the association between the ACE genotype and salt intake interaction with hypertension, so confirmatory studies in other large population-based samples are warranted. The relatively small sample size may cause statistical instability. However, all enrolled participants did not have consanguinity, which is necessary for gene-environment interaction research. Salt intake was assessed by urinary sodium excretion, so it might be underestimated because salt can be removed from the body through other means, for example, sweat. Nevertheless, this sodium excretion estimation method was shown to be closely correlated with the value determined from 24 hours pooled urine. ${ }^{19}$ In addition, because of field conditions, this method was deemed superior to an estimation from a dietary questionnaire.

\section{CONCLUSION}

The interaction of ACE genotype and salt intake on hypertension was observed for both men and women among Chinese Kazakhs, although in different ways. Application comparison of the two models should be of interest to epidemiologists and geneticists for $\mathrm{cG} \times \mathrm{E}$ exploration, and revealed that the GPLTR model might be more suitable to help elucidate the aetiology of complex diseases. Further research is needed to determine the causes for the observed differences behind the interaction mechanisms for men and women.

Acknowledgements We thank the government of Altay City and Hong Dun Town and the Health Bureau of Altay City who supported this project, the village head and staff of each sample village, clinical doctors and nurses of town hospital and people hospital in Altay region, Kazakh college students from Xinjiang Medical University who participated in the surveys, and all participants of this study.

Contributors YW and BZ abstracted data, performed data analysis and interpreted results with substantial contributions from the other co-authors, and drafted the manuscript, which was critically revised by all co-authors. JJ and LH conceived the study and assisted in data analysis, design of figures and tables. WH, FX and YW assisted in data analysis and interpretation, added important background knowledge and improved the manuscript by repeated readings and rephrasing as well as critical discussions of the intellectual content. MZ, YT, SL, WW, KA and CQ contributed to the data collection, quality control, general editing purposes and comments regarding medical terminology and critical review of the manuscript. ZW, $\mathrm{YH}$ and LW mainly performed statistical analyses, designed tables and figures and reviewed the manuscript. All authors have read and approved the final version of this manuscript for submission.
Funding This work was supported by National Natural Science Foundation of China (81273181).

Competing interests None declared.

Patient consent Obtained.

Ethics approval Committee of Institute of the Basic Medical Sciences Chinese Academy of Medical Sciences.

Provenance and peer review Not commissioned; externally peer reviewed.

Data sharing statement № additional data available.

Open Access This is an Open Access article distributed in accordance with the Creative Commons Attribution Non Commercial (CC BY-NC 4.0) license, which permits others to distribute, remix, adapt, build upon this work non-commercially, and license their derivative works on different terms, provided the original work is properly cited and the use is non-commercial. See: http://creativecommons.org/ licenses/by-nc/4.0/

(C) Article author(s) (or their employer(s) unless otherwise stated in the text of the article) 2017. All rights reserved. No commercial use is permitted unless otherwise expressly granted.

\section{REFERENCES}

1. Padmanabhan S, Caulfield M, Dominiczak AF. Genetic and molecular aspects of hypertension. Circ Res 2015;116:937-59.

2. Te Riet L, van Esch JH, Roks AJ, et al. Hypertension: reninangiotensin-aldosterone system alterations. Circ Res 2015;116:960-75

3. Adrogué HJ, Madias NE. Sodium and potassium in the pathogenesis of hypertension. N Engl J Med 2007;356:1966-78.

4. Sayed-Tabatabaei FA, Oostra BA, Isaacs A, et al. ACE polymorphisms. Circ Res 2006;98:1123-33.

5. Ned RM, Yesupriya A, Imperatore G, et al. The ACE I/D polymorphism in US adults: limited evidence of association with hypertension-related traits and sex-specific effects by race/ethnicity. Am J Hypertens 2012;25:209-15.

6. O'Donnell CJ, Lindpaintner K, Larson MG, et al. Evidence for association and genetic linkage of the angiotensin-converting enzyme locus with hypertension and blood pressure in men but not women in the Framingham Heart Study. Circulation 1998;97:1766-72.

7. Ji LD, Zhang LN, Shen P, et al. Association of angiotensinogen gene M235T and angiotensin-converting enzyme gene l/D polymorphisms with essential hypertension in Han Chinese population: a metaanalysis. J Hypertens 2010;28:419-28.

8. Calculator B. Hypertension and the ACE gene. http://www. gbhealthwatch.com/GND-Hypertension-ACE.php (accessed $5 \mathrm{Jul}$ 2016).

9. van der Kleij FG, de Jong PE, Henning $\mathrm{RH}$, et al. Enhanced responses of blood pressure, renal function, and aldosterone to angiotensin I in the DD genotype are blunted by low sodium intake. $J$ Am Soc Nephrol 2002;13:1025-33.

10. Zhang L, Miyaki K, Araki J, et al. Interaction of angiotensin I-converting enzyme insertion-deletion polymorphism and daily salt intake influences hypertension in Japanese men. Hypertens Res 2006;29:751-8.

11. Tabery J. Debating interaction: the history, and an explanation. Int $J$ Epidemiol 2015;44:1117-23.

12. Dick DM, Agrawal A, Keller MC, et al. Candidate gene-environment interaction research: reflections and recommendations. Perspect Psychol Sci 2015;10:37-59.

13. Aliev F, Latendresse SJ, Bacanu SA, et al. Testing for measured gene-environment interaction: problems with the use of crossproduct terms and a regression model reparameterization solution. Behav Genet 2014;44:165-81.

14. Ganzach Y. Misleading interaction and curvilinear terms. Psychol Methods 1997;2:235-47.

15. Mbogning $\mathrm{C}$, Perdry $\mathrm{H}$, Toussile $\mathrm{W}$, et al. A novel tree-based procedure for deciphering the genomic spectrum of clinical disease entities. J Clin Bioinforma 2014;4:1-11.

16. Jiang J, Zhang $B$, Zhang $M$, et al. Prevalence of conventional cardiovascular disease risk factors among Chinese Kazakh individuals of diverse occupational backgrounds in Xinjiang China. Int J Cardiol 2015;179:558-60.

17. Hou L, Zhang M, Han W, et al. Influence of salt Intake on association of blood uric acid with hypertension and related cardiovascular risk. PLoS One 2016;11:e0150451. 
18. Wang W, Weizhong Z, Ninglin S, et al. Chinese guidelines for blood pressure measurement. Chinese Journal of Hypertension 2011;6:1101-15.

19. Kawano $\mathrm{Y}$, Tsuchihashi $\mathrm{T}$, Matsuura $\mathrm{H}$, et al. Report of the Working Group for Dietary Salt Reduction of the Japanese Society of Hypertension: (2) assessment of salt intake in the management of hypertension. Hypertens Res 2007;30:887-93.

20. Wang XF, Wang SZ, Lin RY, et al. [Relationship of I/D polymorphism of angiotensin converting enzyme gene with hypertension in Xinjiang Kazakh isolated group]. Zhonghua Yi Xue Yi Chuan Xue Za Zhi 2003;20:253-5.

21. Zhang B, Simayi A, Sun $Y$, et al. Interaction between genetic and environmental risk factors on essential hypertension in Kazakh Population. J Med Res 2009.

22. Stanković A, Zivković M, Alavantić D. Angiotensin I-converting enzyme gene polymorphism in a serbian population: a genderspecific association with hypertension. Scand J Clin Lab Invest 2002;62:469-75.

23. Chiang FT, Chern TH, Lai ZP, et al. Age- and gender-dependent association of the angiotensin-converting enzyme gene with essential hypertension in a chinese population. J Hum Hypertens 1996;10:823-6.

24. Busjahn A, Knoblauch $\mathrm{H}$, Knoblauch $\mathrm{M}$, et al. Angiotensinconverting enzyme and angiotensinogen gene polymorphisms, plasma levels, cardiac dimensions. A twin study. Hypertension 1997;29:165-70.

25. Krege JH, John SW, Langenbach LL, et al. Male-female differences in fertility and blood pressure in ACE-deficient mice. Nature 1995;375:146-8.
26. Fornage $\mathrm{M}, \mathrm{Amos} \mathrm{Cl}$, Kardia $\mathrm{S}$, et al. Variation in the region of the angiotensin-converting enzyme gene influences interindividual differences in blood pressure levels in young white males. Circulation 1998:97:1773-9.

27. O'Donnell M, Mente A, Yusuf S, et al. And cardiovascular health. Circ Res 2015;116:1046-57.

28. Giner V, Poch E, Bragulat E, et al. Renin-angiotensin system genetic polymorphisms and salt sensitivity in essential hypertension. Hypertension 2000;35:512-7.

29. Caspi A, Sugden K, Moffitt TE, et al. Influence of life stress on depression: moderation by a polymorphism in the $5-\mathrm{HTT}$ gene. Science 2003;301:386-9.

30. Rutter M. Some of the complexities involved in gene-environment interplay. Int J Epidemiol 2015;44:1128-9.

31. Chair MR. Chapter 12 Gene-Environment Interaction in Complex Diseases: Asthma as an Illustrative Case: John Wiley \& Sons, Ltd, 2008:184-97.

32. Keller MC. Gene $x$ environment interaction studies have not properly controlled for potential confounders: the problem and the (simple) solution. Biol Psychiatry 2014;75:18-24.

33. Bastone L, Reilly M, Rader DJ, et al. MDR and PRP: a comparison of methods for high-order genotype-phenotype associations. Hum Hered 2004;58:82-92.

34. Chen J, Yu K, Hsing A, et al. A partially linear tree-based regression model for assessing complex joint gene-gene and gene-environment effects. Genet Epidemiol 2007;31:238-51.

35. Mbogning C, Perdry H, Bagged BP, et al. Tree-based regression procedure for prediction and variable selection. Hum Hered 2015;79:182-93. 